\title{
Eficacia y seguridad de las vacunas contra el SARS-CoV-2
}

\author{
Efficacy and safety of SARS-CoV-2 vaccines
}

\section{Comentado de:}

Fan YJ, et al. Vaccines (Basel). 2021 Sep 4;9(9):989. doi: 10.3390/vaccines9090989. PMID: PMC8473448 ${ }^{1}$

\section{Objetivo}

Evaluar la seguridad y eficacia de las vacunas contra COVID19, comparando el tipo de vacuna y la gravedad del síndrome respiratorio agudo severo generado por el virus SARS-COV-2.

\section{Diseño}

Revisión sistemática y meta-análisis.

\section{Fuentes de Datos}

Los autores realizaron una búsqueda bibliográfica en PubMed y EMBASE. Asimismo, revisaron la sección COVID-19 del sitio ClinicalTrials.gov buscando información sobre estudios en desarrollo.

\section{Selección de estudios}

Incluyeron ensayos clínicos (con información de fase 3 publicada al 30 de mayo de 2021) y estudios observacionales que informaran sobre la seguridad y la eficacia de las vacunas anti COVID-19, sin restricción por idioma, lugar, año de publicación, población, tipo de vacuna o vía de administración. Fueron excluidas las revisiones, las editoriales y los informes no publicados. El artículo no brinda información sobre cuántos autores realizaron la selección, y si lo hicieron o no de manera independiente.

\section{Extracción de datos}

Los datos extraidos incluyeron los detalles de la publicación, el diseño, el nombre de la vacuna, el tipo de plataforma (tales como ARNm, vectores virales, virus inactivado) y vía de administración, el número de dosis, la población del estudio, el número de personas que sufrieron eventos adversos y el tipo de eventos, el número de casos de COVID-19 (sintomáticos o asintomáticos) detectados, la severidad de la infección por SARS-CoV-2 en los grupos control y vacunados. La calidad de cada estudio fue evaluada utilizando la herramienta QUIPS (Quality in Prognostic Studies). EI articulo no especifica cuántos autores realizaron la extracción de datos, y si lo hicieron o no de manera independiente.

\section{Medidas de desenlace}

El desenlace principal de seguridad fue la incidencia de eventos adversos serios (EAS) reportada luego de recibir al menos una dosis de vacuna, empleando razones de probabilidad para la comparación de los grupos (odds ratio [OR]).

Los desenlaces de eficacia fueron la incidencia de COVID-19 luego de una o dos dosis de vacuna, de casos sintomáticos y asintomáticos ocurridos luego de dos dosis, y la severidad de las infecciones por SARS-CoV-2 desarrolladas luego de tener un esquema de vacunación completo, empleando riesgos relativos (RR) para la síntesis cuantitativa.

\section{Resultados principales}

Los autores incluyeron 12 estudios de vacunas para COVID-19 (ocho ensayos clínicos en fase III y cuatro estudios observacionales), de los cuales seis fueron incluidos en el meta-análisis de seguridad, seis en el de eficacia luego de una dosis de vacuna, y diez, en el de eficacia luego de dos dosis. La Tabla 1 resume los principales resultados.

En términos de seguridad, las vacunas de ARNm mostraron más EAS que las vacunas de vectores virales y las inactivadas, pero no hubo evidencia sólida que indicase que las vacunas COVID-19 causasen de manera directa esos eventos, como queda evidenciado por la amplitud de los intervalos de confianza (Tabla 1). Los trastornos metabólicos, musculoesqueléticos, inmunológicos y renales graves fueron más comunes entre los receptores de vacunas inactivadas, y las complicaciones gastrointestinales graves fueron más frecuentes entre los receptores de vacunas inactivadas y de vectores virales. La aparición de trastornos vasculares serios fue más frecuente en las vacunas de ARNm.

Al analizar de manera global la eficacia de dos dosis de cualquiera de las vacunas, los autores informaron una reducción global del riesgo de presentar COVID-19 (RR 0,16, IC 95\% 0,10 a 0,25$)$, que fue mayor para la prevención de casos sintomáticos (RR 0,11, IC $95 \% 0,04$ a 0,30) que asintomáticos (RR 0,34 IC $95 \% 0,08$ a 1,56). Asimismo, la reducción del riesgo relativo (RRR) fue de $88 \%$ (IC 95\% $70 \%$ a $95 \%$ ) para la infección severa por SARS-CoV- 2, $92 \%$ (IC $95 \% 76 \%$ a $98 \%$ ) para la hospitalización por esta enfermedad, y $86 \%$ (IC $95 \% 31 \%$ a $97 \%$ ) para el riesgo de muerte.

Las vacunas que usaron plataformas de ARNm fueron más eficaces para reducir los números de casos de COVID-19 que los otros tipos de vacunas luego de dos dosis (Tabla 1 ).

Tabla 1. Resultados principales de la seguridad y la eficacia de las vacunas contra el SARS-CoV-2 clasificadas según el tipo de plataforma y en forma global, en comparación con placebo. Nota: IC: intervalo de confianza; OR: odds ratio; RR: riesgo relativo.

\begin{tabular}{|l|l|l|l|}
\hline Desenlace & \multicolumn{1}{|c|}{ ARNm } & \multicolumn{1}{c|}{$\begin{array}{c}\text { Vectores virales } \\
\text { no-replicativos }\end{array}$} & Inactivadas \\
\hline Eventos adversos serios, OR (IC 95\%) & $1,47(0,65$ a 3,29) & $0,76(0,62$ a 0,93$)$ & $0,79(0,62$ a 1,00$)$ \\
\hline $\begin{array}{l}\text { Incidencia de COVID-19 con dos dosis, } \\
\text { RR (IC 95\%) }\end{array}$ & $0,05(0,02$ a 0,13$)$ & $0,33(0,22$ a 0,50$)$ & $0,32(0,23$ a 0,42$)$ \\
\hline $\begin{array}{l}\text { Incidencia de COVID-19 con una dosis, } \\
\text { RR (IC 95\%) }\end{array}$ & $0,53(0,23$ a 1,20$)$ & $0,29(0,11$ a 0,76$)$ & No informado \\
\hline
\end{tabular}




\section{Conclusiones}

Las vacunas COVID-19 evaluadas en esta revisión son seguras y efectivas. Los resultados indican que dos dosis de vacuna de ARNm podrían prevenir la infección por SARS-COV-2 de manera más eficaz y asociarse a más EAS, pero se necesitan más investigaciones debido al alto grado de heterogeneidad entre los estudios. Considerando que la mayor eficacia se observó al recibir dos dosis de vacuna y en la prevención de infecciones sintomáticas (y en menor medida, las asintomáticas), los autores recomiendan continuar con las medidas de protección, como el uso de barbijos y el distanciamiento social, aun después de haber recibido una o dos dosis de vacunas.

Fuente de financiamiento / Conflicto de interés de los autores: La investigación no recibió financiamiento externo y los autores declararon no tener conflicto de intereses.

\section{Comentario}

Esta revisión ${ }^{1}$ mostró que la vacunación completa contra COVID-19 protege a la mayoría de las personas frente al SARS COV-2 y, lo que es aún más importante, reduce las formas severas y la muerte por COVID-19. La evidencia en cuanto a EAS es insuficiente, pues los efectos estimados fueron imprecisos y heterogéneos. Por lo tanto, se necesitan más investigaciones para conocer la magnitud exacta y las diferencias entre las vacunas con respecto a su seguridad y eficacia. Sin embargo, la experiencia global, con más de 6.900 millones de dosis administradas ${ }^{2}$, parece indicar que las vacunas COVID-19 son seguras.

La investigación emergente en este tema es desbordante. Sólo en la plataforma L.OVE (Living Overview of the Evidence) de búsqueda de revisiones sistemáticas se encuentran, a la fecha de escribir este comentario, 137 revisiones sistemáticas. Es por eso que para temas de tanta actualidad y dinamismo editorial, son sumamente importantes las revisiones sistemáticas vivas (RSV) que realizan búsquedas y actualizaciones periódicas de la evidencia acumulada.

Con un grupo colaborativo internacional ${ }^{3}$ pre-publicamos un primer reporte de una RSV, analizada mediante meta-análisis en red, que permite incorporar la evidencia de todas las comparaciones y mejorar la precisión de las estimaciones ${ }^{4}$. Nos enfocamos sólo en ensayos clínicos aleatorizados (ECA) para determinar su eficacia e incluimos 22 de ellos al 12 de mayo de 2021. Encontramos una eficacia en la prevención de COVID-19 de 95\% (IC 95\% $92 \%$ a $97 \%$ ) con las vacunas de ARNm (moderada certeza de evidencia GRADE $^{5}$ y de $68 \%$ (IC $95 \% 61 \%$ a $74 \%$ ) con vacunas de vectores virales (baja certeza de evidencia). Las vacunas con vectores virales redujeron la mortalidad $75 \%$ (IC $95 \% 33 \%$ a $91 \%$, baja certeza de evidencia), pero los datos de mortalidad sobre las vacunas de ARNm eran aún imprecisos. Ninguna de las vacunas mostró evidencia de una diferencia en la incidencia de EAS, pero la evidencia observacional indicó que éstos eran raros. Tanto las vacunas de ARNm como las de vectores virales aumentaron el riesgo de EA no serios. En términos generales, los resultados fueron consistentes con la revisión de Fan et al ${ }^{1}$ en tanto las vacunas de ARNm parecen ser más efectivas para prevenir la COVID-19, pero encontramos que las vacunas de vectores virales parecen ser más efectivas para reducir la mortalidad.

Otra RSV 6 incluyó 30 estudios mayoritariamente observacionales (sólo dos eran ECA) a mayo de 2021. Los estudios se realizaron en diferentes países (Dinamarca, Israel, Italia, Qatar, España, Suecia, Reino Unido, EE.UU.). El rango de efectividad en la prevención de infecciones con una dosis de vacuna osciló entre el 16,9\% y el 91,2\% (en su mayoría, entre el $60 \%$ y el $70 \%$ ). El rango de efectividad con dos dosis de vacuna osciló entre el $61,7 \%$ y el $98,6 \%$ (la mayoría, entre el $80 \%$ y el $90 \%$ ). Las estimaciones no se vieron afectadas por el tipo de vacuna o el diseño del estudio. La efectividad contra la infección asintomática después de una dosis de una dosis osciló entre el $36 \%$ y el $79 \%$ y con dos dosis, entre el $80 \%$ y el $94 \%$.

\section{Conclusiones del comentador}

Desde una perspectiva de salud pública, se puede concluir que las personas completamente vacunadas obtienen una altísima protección contra las formas serias de COVID-19, y también para infecciones no graves por SARS-CoV-2, incluidas las asintomáticas. La mayoría de las vacunas tienen eficacia y efectividad comparables. Considerando el tiempo récord en que se desarrollaron las vacunas, estos hallazgos, provenientes tanto de investigaciones experimentales como observacionales, suponen un enorme impacto poblacional y en los sistemas de salud, reduciendo la transmisión y las complicaciones del SARS-CoV-2, aunque se desconoce con exactitud su efecto según la cepa dominante. De manera desafortunada, estos beneficios los están recibiendo principalmente los países de mejores ingresos, lo que desnuda una inequidad de escala universal. No se ha comprendido aún que el abordaje debe ser global, no solamente por cuestiones éticas ineludibles, sino también por cuestiones prácticas. En la medida que persistan reservorios humanos para el virus, existen posibilidades de que nuevas variantes del SARS-CoV-2 amenacen todos los logros conseguidos. Por otro lado, puede resultar obscena la aplicación de terceras dosis en algunos países, existiendo aún una enorme población no inmunizada en el resto del mundo. Sólo mediante la vacunación masiva contra el virus SARS-CoV-2, equitativamente distribuida, será posible acabar con esta pandemia mundial.

Agustín Ciapponi [ Hospital Italiano de Buenos Aires, Servicio de Medicina Familiar y Comunitaria; Centro Cochrane Argentina; Instituto de Efectividad Clínica y Sanitaria. aciapponi@iecs.org.ar ]

Ciapponi A. Eficacia y seguridad de las vacunas contra el SARS-CoV-2. Evid Actual Pract Ambul. 2021;24(4):e002162. Available from: https://dx.doi.org/ 10.51987/EVIDENCIA.V25I1.6981. Comentado de: Fan YJ, et al. Safety and Efficacy of COVID-19 Vaccines: A Systematic Review and Meta-Analysis of Different Vaccines at Phase 3. Vaccines (Basel). 2021 Sep 4;9(9):989. doi: 10.3390/vaccines9090989. PMID: 34579226 


\section{Referencias}

1. Fan YJ, Chan KH, Hung IFN. Safety and Efficacy of COVID-19 Vaccines: A Systematic Review and Meta-Analysis of Different Vaccines at Phase 3. Vaccines (Basel). 2021;9(9):989. Available from: 10.3390/vaccines9090989.

2. Coronavirus Resource Center, Johns Hopkins University. COVID-19 Dashboard;. Available from: https://coronavirus.jhu.edu/map.html [Last access: 2021-10-29].

3. Korang SK, von Rohden E, Veroniki AA, et al.. Vaccines to Prevent COVID-19: A Living Systematic Review With Trial Sequential Analysis and Network Meta-Analysis of Randomised Controlled Trials; 2021. Available from: 10.2139/ssrn.3854588.

4. Ciapponi A. La ola de los meta-análisis en red: una herramienta cada vez más útil para la toma de decisiones. Evid Actual Pract Ambul. 2018;21(4):98-99. Available from: 10.51987/evidencia.v21i4.6853.

5. Guyatt G, Oxman AD, Akl EA, et al. GRADE guidelines: 1. Introduction-GRADE evidence profiles and summary of findings tables. J Clin Epidemiol. 2011;64(4):383-394. Available from: 10.1016/j.jclinepi.2010.04.026.

6. Harder T, Koch J, Vygen-Bonnet S, et al. Efficacy and effectiveness of COVID-19 vaccines against SARS-CoV-2 infection: interim results of a living systematic review, 1 January to 14 May 2021. Euro Surveill. 2021;26(28):2100563. Available from: 10.2807/1560-7917.ES.2021.26.28.2100563. 